\title{
La restauración ecológica como estrategia de construcción social en la Vereda Chipautá, Municipio de Guaduas, Cundinamarca*
}

Ecological Restoration as a Social Construction Strategy in the Chipautá District, Town of Guaduas, Cundinamarca

Rosa Catalina Hernández-Gómez ${ }^{\mathrm{a}}$

Universidad Distrital Francisco José de Caldas, Colombia

ORCID: http://orcid.org/0000-0002-1287-5707

Edgard Cantillo-Higuera

Universidad Distrital Francisco José de Caldas, Colombia

ORCID: http://orcid.org/0000-0002-5537-2413
DOI: https://doi.org/10.11144/Javeriana.ayd22-42.reec Redalyc: http://www.redalyc.org/articulo.oa? id $=151557418005$

Fecha de recepción: 18 Julio 2017 Fecha de publicación: 30 Junio 2018

\section{Resumen:}

Esta investigación propone un modelo metodológico de restauración ecológica como una estrategia de construcción social, en la vereda Chipautá, Guaduas. Se definieron cuatro etapas, en la primera se obtuvieron las bases teóricas necesarias para la construcción del modelo, en la segunda se recolectaron datos en campo mediante las técnicas cartografía social, entrevistas semiestructuradas, identificación de problemas y reunión con grupos sociales, y se realizó una revisión sistemática de bibliografía de experiencias de restauración ecológica con enfoque social, la cual permitió obtener elementos estructurantes que aportaron al modelo. En la tercera etapa se interpretaron los resultados y se validaron mediante triangulación de datos. Finalmente, se presentó el modelo metodológico. Con el modelo propuesto, se logró contribuir al campo de conocimiento en restauración ecológica, planteando un nuevo marco de análisis que permita recuperar los ecosistemas degradados y proponer alternativas de desarrollo sustentable y de manejo adaptativo ante los cambios ambientales actuales en las comunidades involucradas.

Palabras clave: transformación social, restauración ecológica, construcción social, sistemas socio-ecológicos.

\begin{abstract}
:
This research proposes a methodological model of ecological restoration as a strategy for the social construction in the district Chipautá, town of Guaduas. Four stages were defined: the first stage set the theoretical grounds required to build the model. The second stage consisted in the data collection out in the field with social cartographic techniques, semi-structured interviews, problem identification and meetings with social groups. It also included a systematic review of the bibliography dealing with ecological restoration experiences under a social approach, which allowed to obtain structuring elements that would contribute to the model. The third stage was intended to interpret and validate the results through the data triangulation. The final stage was the presentation of the methodological model itself. The proposed model contributed to the knowledge field of ecological restoration by setting out a new analytical framework enabling to recover degraded ecosystems and propose alternatives of both sustainable development and adaptative management to cope with the current environmental changes in the involved communities.
\end{abstract}

Keywords: social transformation, ecological restoration, social construction, social-ecological systems.

\section{Introducción}

La restauración ecológica tiene como objetivo la recuperación de los ecosistemas perturbados con el fin de revertir su deterioro y restablecer su integridad ecológica (Keenleyside et al., 2014). Según la Sociedad para la Restauración Ecológica (SER), la restauración ecológica es definida como "el proceso de asistencia a la recuperación de un ecosistema que ha sido degradado, dañado o destruido”. Tal definición implica un esfuerzo práctico para recuperar de forma asistida las dinámicas naturales de los ecosistemas que se desean restaurar, teniendo presente que lo que se busca no es dejar el ecosistema como se encontraba antes del disturbio, sino recuperar los componentes básicos de su estructura, función y composición, que le faciliten desarrollarse y mantenerse en el tiempo (Román, Levy, Aguirre, \& Sánchez, 2011).

En la actualidad, existen metodologías propuestas (Hallett, Hobbs, \& Mooney, 2011; Barrera-Cataño, Contreras-Rodríguez \& Garcón-Yepes, 2010; Cantillo, Lozada, \& Pinzón, 2009; Ritchlin, 2001; Clewell, 
Rieger, \& Munro, 2000) que sirven como recomendaciones generales para desarrollar procesos de restauración ecológica; sin embargo, en estas, la inclusión y el estudio de la dimensión social del área objeto de restauración, no es considerada. Las poblaciones locales desempeñan un papel netamente participativo en ciertas etapas del proceso y es limitado, por una parte, a brindar información que alimente el diagnóstico inicial; y por otra, a realizar un acompañamiento en ciertas actividades proyectadas por el equipo restaurador.

Existen, a su vez, planteamientos a nivel internacional donde se han establecido directrices que buscan una mayor planificación y organización para la efectividad de los procesos de restauración ecológica. Las directrices de la Organización Internacional de las Maderas Tropicales (OIMT, 2002) para la restauración, ordenación y rehabilitación de bosques tropicales secundarios y degradados enfatizan en la necesidad de involucrar en la planificación de la restauración, aspectos políticos y condiciones jurídicas y sociales existentes dentro y fuera del área de estudio. Señalan, además, que existen actores que tienen un interés en el ecosistema y, por tanto, los esfuerzos de restauración, ordenación o rehabilitación deben realizarse con su plena participación. De forma similar, Gann y Lamb (2006), de la mano con la SER y la Unión internacional para la conservación de la naturaleza (UICN), van más allá y proponen catorce (14) principios de la buena práctica de la restauración ecológica, en los cuales se integran el sistema ecológico y el humano (ver figura 1).

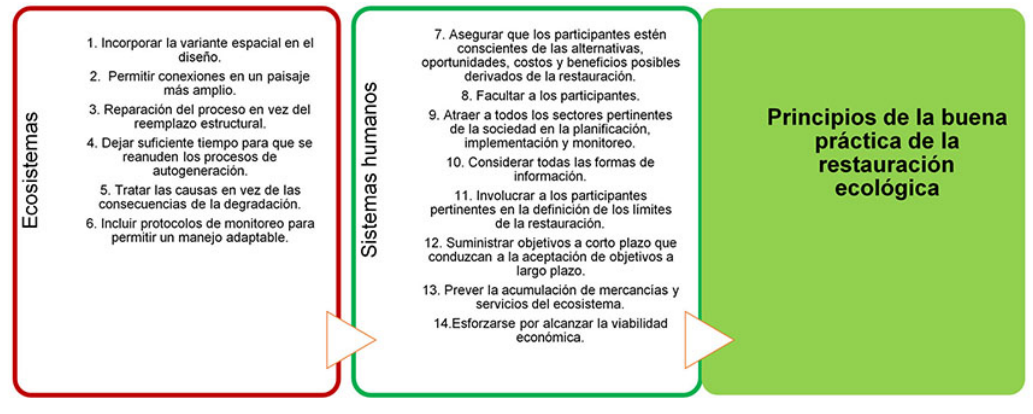

FIGURA 1.

Principios de la buena práctica de la restauración ecológica.

Fuente: modificado de Gann y Lamb, 2006

A pesar de lo anterior, aún no existe un modelo que integre los procesos de restauración ecológica y los sistemas socio-ecológicos (SSE), lo que permitiría tener una visión integrada de las relaciones existentes en el territorio. Es importante comprender la multidisciplinariedad del proceso y la necesidad de abordarlo desde un enfoque integral y multidimensional que considere los componentes sociales, económicos, políticos, culturales y ecológicos, lo cual contribuiría a desarrollar procesos de restauración ecológica más acorde con la realidad de la región y de sus habitantes, y orientado a la formulación de alternativas de desarrollo sustentable para las poblaciones.

Dentro del marco de análisis de los SSE, la restauración ecológica debe ser vista como un proceso complejo que involucra una amplia gama de factores, tanto naturales como sociales, que depende de las metas y estrategias de restauración establecidas, las cuales serán distintas considerando el ecosistema y su historia de transformación; inclusive, los procesos de restauración pueden ser diferentes en distintos sitios del ecosistema.

La formulación del modelo teórico - metodológico de restauración ecológica que se plantea en la presente investigación, busca, además de la recuperación ecosistémica, una transformación social que permita la reconfiguración de la relación sociedad-naturaleza entre los habitantes de la vereda Chipautá y alrededores, frente a la reserva forestal, que se traduzca en una construcción social del proceso de restauración ecológica que lleve a alternativas de desarrollo sustentable para la comunidad.

Adicionalmente, el modelo propuesto en la vereda Chipautá pretende instaurar un nuevo enfoque metodológico orientado a incluir en la restauración ecológica el análisis de los sistemas socioecológicos (SSE) en su totalidad como elemento articulador de la relación sociedad-naturaleza, y la inclusión permanente de las comunidades involucradas, no sólo con propósitos participativos, sino convirtiéndolos en actores intervinientes que inicien procesos de transformación que lleven a la construcción social de la restauración ecológica en la población (Barkin, 2012) 


\section{Metodología}

\section{Descripción del área de estudio}

La vereda Chipautá se encuentra localizada en el municipio de Guaduas, en el departamento de Cundinamarca, Colombia, y hace parte de la provincia bajo Magdalena, a una altitud de $1695 \mathrm{~m}$ s.n.m. Cuenta con una superficie veredal de 464,25 ha, lo que corresponde al 16,11\% del municipio. Limita al norte con las veredas el Palmar y San José; al sur, con las veredas Raizal-Cajón y Granada; al este, con la vereda la Carbonera; y al oeste, con las veredas San Isidro y La Verbena del municipio de Quebradanegra (ver figura 2).

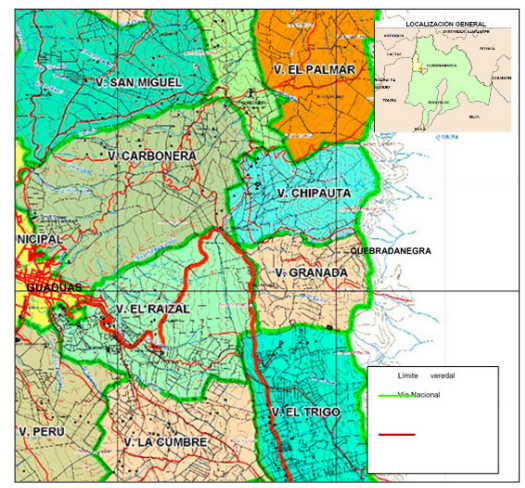

FIGURA 2.

Localización general de la Vereda Chipautá, Guaduas.

Fuente: Secretaría de Planeación Guaduas (SPG), 2009

La Vereda Chipautá, a su vez, se encuentra localizada dentro de la Reserva Forestal Protectora Cuenca del Río San Francisco, declarada, mediante el Acuerdo 038 del 27 de octubre de 1981 del Instituto Nacional de los Recursos Naturales Renovables y del Ambiente (Inderena), y la Resolución Minagricultura 242 del 30 de septiembre de 1983, con una extensión de 2200 ha. Posteriormente, mediante el Acuerdo Inderena $\mathrm{N}^{\circ} 62$ del 23 de septiembre de 1985, y la Resolución Minagricultura No. 01 de 1986, se amplió la zona en 680 ha, para un total de 2880 ha (Parques Nacionales Naturales de Colombia [PNN], 2009), la cual fue establecida teniendo en cuenta la importancia hídrica que tiene la zona para el municipio de Guaduas.

\section{Enfoque metodológico}

La presente investigación se basa en un enfoque mixto, en el cual se analizan datos cualitativos y cuantitativos en un mismo estudio con el fin de resolver un problema de investigación (Hernández, Fernández, y Baptista, 2004). En este sentido, se tomaron datos mediante diversos métodos de recolección de información para identificar los principios que sustenten un modelo metodológico de restauración ecológica, que integre los aspectos sociales a las técnicas propias del proceso y que logre una construcción social de la restauración ecológica en los actores sociales identificados en la vereda Chipautá (Guaduas, Cundinamarca).

\section{Contexto de la investigación}

La investigación se desarrolla en el departamento de Cundinamarca, en el municipio de Guaduas, en la vereda Chipautá, donde se lleva a cabo un programa de reforestación en la parte alta de la vereda que se denomina Reserva Hídrica Chipautá. La investigadora, en compañía del Semillero de Investigación en Restauración Ecológica (SIRE) de la Universidad Distrital Francisco José de Caldas, se reunió con los diferentes actores de 
la comunidad, como los miembros de la Junta de Acción Comunal de la Vereda Chipautá, miembros de la comunidad en general, la Alcaldía Municipal, la Corporación Autónoma Regional de Cundinamarca (CAR), las Unidades Municipales de Asistencia Técnica Agropecuaria (Umata) y los docentes de la escuela veredal. Con el fin de dar inicio a las labores investigativas, y a lo largo del proyecto, se mantuvo en contacto con los diferentes actores para la recolección y análisis de la información necesaria para el presente estudio.

\section{Etapas de la investigación}

En la investigación se definieron cuatro (4) etapas con el fin de recolectar, analizar e interpretar los datos y proponer, finalmente, el modelo metodológico (figura 3).

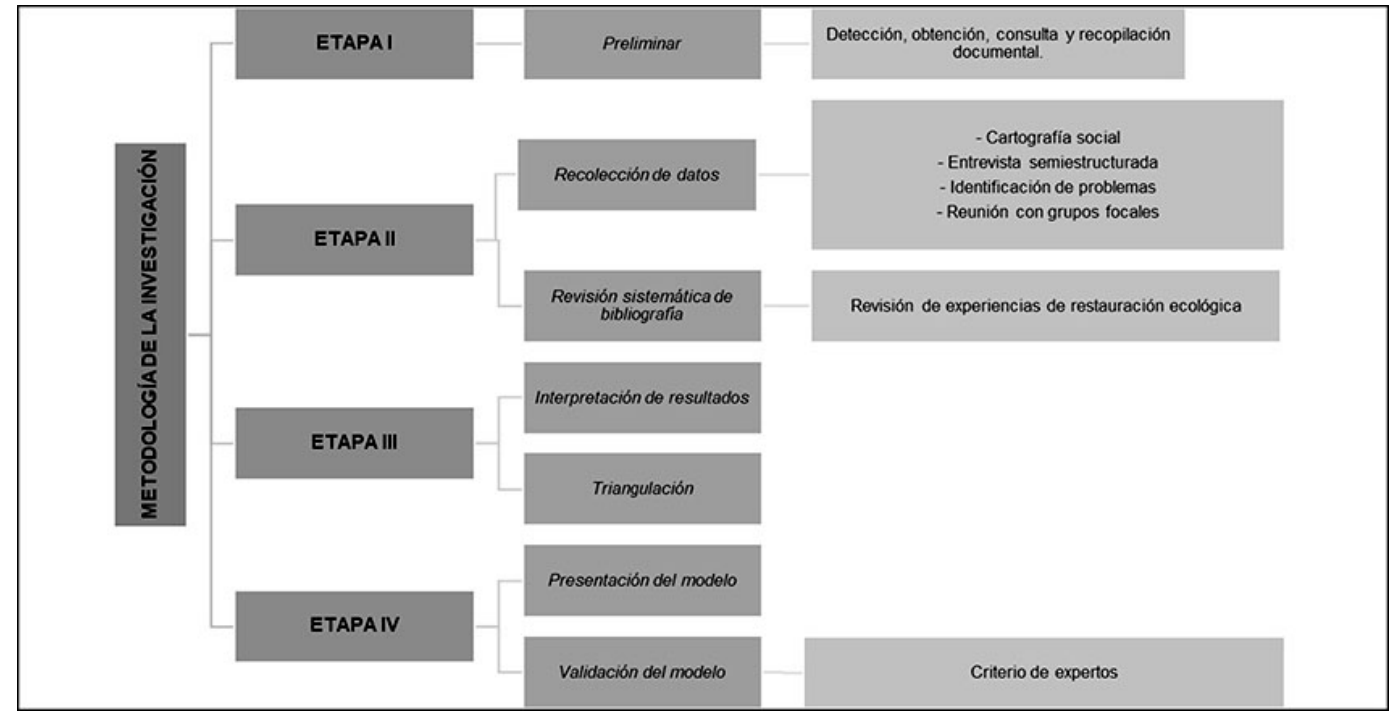

FIGURA 3 .

Metodología de la investigación.

Fuente: elaboración propia.

\section{Resultados y análisis}

\section{Recolección de datos}

De acuerdo con las técnicas utilizadas y la información obtenida, se realizó una triangulación que consiste en la verificación y comparación de los datos procedentes de las diversas fuentes de recolección utilizadas, con el fin de validar los datos obtenidos, además de complementar descriptivamente la información adquirida con las técnicas del enfoque mixto. Los datos que se triangulan provienen de la cartografía social, las entrevistas semiestructuradas y los grupos focales.

A continuación, se muestra la triangulación de los datos teniendo las siguientes categorías de análisis:

- Expectativas y necesidades de la población local frente a los recursos naturales presentes en la vereda. 
TABLA 1 .

Triangulación expectativas y necesidades de la población local

\begin{tabular}{|c|c|c|}
\hline Cartografía social & $\begin{array}{l}\text { Entrevistas } \\
\text { semiestructuradas }\end{array}$ & Grupos focales \\
\hline $\begin{array}{l}\text { La población percibe en un } \\
\text { escenario futuro que las zonas } \\
\text { boscosas se incrementarán } \\
\text { desde la parte alta de la } \\
\text { vereda. Además, enfatiza en la } \\
\text { protección y conservación del } \\
\text { recurso hídrico debido al } \\
\text { servicio ambiental que presta. }\end{array}$ & $\begin{array}{l}\text { Se proyecta un incremento } \\
\text { en cuanto a la calidad y la } \\
\text { cantidad del recurso hídrico } \\
\text { debido a la protección de la } \\
\text { Reserva Hídrica Chipautá, } \\
\text { ubicada en la parte alta, ya } \\
\text { que consideran que la } \\
\text { cobertura boscosa tiende a } \\
\text { aumentar gracias a los } \\
\text { proyectos y actividades que } \\
\text { se vienen adelantando. }\end{array}$ & $\begin{array}{l}\text { Los participantes } \\
\text { manifiestan, de acuerdo con } \\
\text { la propuesta de planificación } \\
\text { biorregional, que las áreas } \\
\text { centrales corresponden a la } \\
\text { parte alta de la vereda, ya } \\
\text { que nacen las Quebradas } \\
\text { Llanadas y Ventas que } \\
\text { aportan la mayor parte del } \\
\text { caudal al río San Francisco. }\end{array}$ \\
\hline
\end{tabular}

Fuente: elaboración propia

Por una parte, existe una evidente concordancia entre los datos provenientes de la cartografía social, la entrevista semiestructurada y los grupos focales, ya que se observa que en todas las fuentes la comunidad mantiene una idea común de que los recursos bosque y agua deben ser conservados, y que tienden a aumentar en un escenario futuro. Tales datos sirven de base para orientar las labores de restauración ecológica a la protección y suministro del recurso hídrico en la vereda (ver tabla 1). Por otra, esta información muestra la necesidad de un diagnóstico integrado (general y participativo) del área de estudio con el fin de complementar las percepciones de la población respecto a sus recursos naturales, y debe ir acompañada de la construcción de conocimiento del entorno natural y su dinámica interna y externa.

- Tendencias de transformación ambiental en la zona.

TABLA 2 .

Triangulación tendencias de transformación ambiental en la zona

\begin{tabular}{|c|c|c|}
\hline Cartografía social & $\begin{array}{l}\text { Entrevistas } \\
\text { semiestructuradas }\end{array}$ & Grupos focales \\
\hline $\begin{array}{l}\text { La transformación } \\
\text { ambiental tiene una } \\
\text { tendencia a la } \\
\text { expansión de las áreas } \\
\text { boscosas con miras a } \\
\text { impulsar el ecoturismo } \\
\text { en la vereda. En un } \\
\text { escenario futuro se } \\
\text { presentarán procesos de } \\
\text { reemplazo de las } \\
\text { actividades agrícolas } \\
\text { por las ganaderas. }\end{array}$ & $\begin{array}{l}\text { El } 75 \% \text { de los } \\
\text { entrevistados manifiesta } \\
\text { que el futuro de la } \\
\text { actividad agrícola es } \\
\text { desalentador, ya que } \\
\text { tiende a desaparecer al ser } \\
\text { estas áreas reemplazadas } \\
\text { para ganado; no obstante, } \\
\text { refieren que en la parte } \\
\text { alta se incrementará el } \\
\text { uso forestal para la } \\
\text { protección del recurso } \\
\text { hídrico. }\end{array}$ & $\begin{array}{l}\text { La transformación del territorio es } \\
\text { percibida por los grupos focales como } \\
\text { uno de los elementos más importantes } \\
\text { a tener en cuenta, dado que consideran } \\
\text { que esta se está realizando de manera } \\
\text { desordenada sin considerar las áreas a } \\
\text { conservar, por el acelerado grado de } \\
\text { transformación y la conversión de } \\
\text { áreas agrícolas a ganaderas; lo que } \\
\text { también concuerda con la } \\
\text { identificación de problemas referente } \\
\text { a la tala de árboles para uso agrícola, } \\
\text { que se considera un problema focal. }\end{array}$ \\
\hline
\end{tabular}

Fuente: elaboración propia 
La tendencia de transformación ambiental en la vereda se encuentra permeada por la dinámica social existente en el territorio, lo que evidencia concordancia en un escenario futuro donde predominará el uso forestal en la parte alta, con miras a la instauración de actividades ecoturísticas; mientras que en la parte baja se augura un reemplazo de las actividades agrícolas por las ganaderas, debido a la baja productividad del suelo y los cambios en la estructura económica del municipio (ver tabla 2). Estos resultados muestran la importancia de una planificación ambiental territorial de la vereda con el fin de ordenar el territorio y seleccionar las áreas a restaurar, y determinar zonas de recuperación y zonas productivas de acuerdo con la potencialidad del terreno. De igual forma, resulta necesaria la construcción del conocimiento comunitario referente a las características ambientales del territorio y formas de alianzas estratégicas que articulen a la comunidad y a las entidades locales para promover estrategias de desarrollo sustentable acordes con los procesos de restauración ecológica y de reforestación que se vienen desarrollando.

- Potencialidades ambientales reconocidas por la población local, específicamente bienes y servicios ambientales.

TABLA 3 .

Triangulación potencialidades ambientales reconocidas por la población local

\begin{tabular}{|c|c|c|}
\hline Cartografía social & $\begin{array}{l}\text { as } \\
\text { cturadas }\end{array}$ & Grupos focales \\
\hline $\begin{array}{l}\text { La comunidad percibe que } \\
\text { la potencialidad ambiental } \\
\text { de la vereda es el recurso } \\
\text { hídrico, el cual se } \\
\text { materializa a nivel } \\
\text { municipal, debido al aporte } \\
\text { que presentan las quebradas } \\
\text { que nacen en la parte alta. El } \\
\text { componente paisajístico es } \\
\text { también determinante para } \\
\text { la comunidad, considerando } \\
\text { el proyecto ecoturístico que } \\
\text { han venido desarrollando. } \\
\text { De igual, manera, los } \\
\text { servicios de provisión que } \\
\text { presta el territorio son } \\
\text { evidenciados en las } \\
\text { actividades agrícolas y } \\
\text { ganaderas que sostienen a la } \\
\text { población. }\end{array}$ & $\begin{array}{l}\text { La población considera que lo } \\
\text { primordial es la conservación } \\
\text { del recurso hídrico, ya que } \\
\text { constituye el principal } \\
\text { servicio ambiental que provee } \\
\text { el ecosistema, lo cual se ve } \\
\text { reflejado en los esfuerzos de } \\
\text { la población por recuperar la } \\
\text { cárcava que altera la cantidad } \\
\text { y calidad de este recurso. } \\
\text { Además, la tendencia a la } \\
\text { ampliación de las áreas } \\
\text { boscosas, en un escenario } \\
\text { presente y futuro, confirma la } \\
\text { importancia del bosque y sus } \\
\text { elementos para las actividades } \\
\text { de educación ambiental y } \\
\text { ecoturística. }\end{array}$ & $\begin{array}{l}\text { Considerando la propuesta de } \\
\text { planificación biorregional } \\
\text { efectuada por la población, en } \\
\text { la cual señalan que el } \\
\text { ordenamiento ambiental en la } \\
\text { vereda debe realizarse en } \\
\text { torno al recurso hídrico, } \\
\text { representando como áreas } \\
\text { centrales las zonas de } \\
\text { nacimiento de cuerpos de } \\
\text { aguas con el fin de mantener } \\
\text { o mejorar las condiciones de } \\
\text { calidad y cantidad del } \\
\text { recurso. Las actividades } \\
\text { productivas al interior de la } \\
\text { vereda ocupan un } \\
\text { componente importante para } \\
\text { la población como impulsores } \\
\text { económicos en la actualidad. }\end{array}$ \\
\hline
\end{tabular}

Fuente: elaboración propia.

En este aspecto, se muestra una concordancia en que los bienes y servicios más importantes en la vereda Chipautá, y que son objeto de conservación, son el recurso hídrico y el bosque, lo que los convierte en factores integradores de la restauración ecológica a la dinámica social de la vereda (ver tabla 3). Tener una visión homogénea del territorio entorno a su manejo es un paso importante para la instauración de proyectos de rehabilitación ambiental. Además, es nuevamente evidente la necesidad de un diagnóstico integrado y una planificación territorial para la formulación de proyectos ambientales y una discusión entre los diferentes 
actores sociales sobre las estrategias de desarrollo local y sustentable de acuerdo con las potencialidades del territorio.

\section{Revisión sistemática de bibliografía}

Se realizó una revisión sistemática de la literatura de restauración ecológica con un enfoque social, tomando como punto de partida las bases de datos ScienceDirect, Scopus y Google Académico, con el fin de extraer elementos reales de experiencias de restauración ecológica que incluyen a la comunidad, o con iniciativa de esta, y que hayan logrado de alguna forma una transformación social para, de esta manera, encontrar factores determinantes en los procesos de restauración ecológica. Después de una serie de filtros realizados a los 296 artículos revisados, se obtuvieron 74 artículos y ocho libros para el análisis (ver tabla 4).

TABLA 4 .

Algunas experiencias de restauración ecológica revisadas

\begin{tabular}{|c|c|c|c|}
\hline \multirow{2}{*}{ Artículo } & \multicolumn{2}{|c|}{ Evidencia de transformación social } & \multirow{2}{*}{ Bibliografía } \\
\hline & Estrategia de restauración & Beneficio económico y social & \\
\hline $\begin{array}{l}\text { Restauración ecológica de } \\
\text { ecosistemas de Humedales } \\
\text { Bangladesh, Dhaka }\end{array}$ & $\begin{array}{l}\text { Restauración enfocada a humedales, } \\
\text { conectando llanuras de inundación con } \\
\text { los ríos locales a través de canales. }\end{array}$ & $\begin{array}{c}\text { La producción de peces aumentó cinco veces la } \\
\text { producción inicial, lo cual permitió la reactivación de la } \\
\text { economía ribereña, fortaleciendo el tejido social que se } \\
\text { deriva de la pesca. }\end{array}$ & $\begin{array}{l}\text { Rahman y Minkin, } \\
2003\end{array}$ \\
\hline $\begin{array}{l}\text { Gestión de la reforestación del } \\
\text { Bosque Senegal, Africa }\end{array}$ & $\begin{array}{l}\text { Aumentar la cobertura vegetal y usar } \\
\text { técnicas de conservación de suelo para } \\
\text { prevenir la erosión. }\end{array}$ & $\begin{array}{l}\text { La fauna silvestre volvió al bosque, y se incentivó el } \\
\text { ecoturismo, trayendo beneficios económicos a la } \\
\text { población, donde sus ingresos aumentaron en un 30\% y } \\
\text { por consiguiente aumentó su calidad de vida. }\end{array}$ & Ndiaye, 2003 \\
\hline $\begin{array}{l}\text { Manejo y restauración comunitaria } \\
\text { en el Bosque de Igbodja, África }\end{array}$ & $\begin{array}{l}\text { Se reforestaron cuatro poblados con } \\
\text { especies nativas, con el fin de restablecer } \\
\text { el ecosistema. Se incentivó la apicultura y } \\
\text { se realizaron acciones normativas para } \\
\text { instaurar una ley forestal en la región. }\end{array}$ & $\begin{array}{l}\text { El proyecto permitió establecer otras alternativas de } \\
\text { gestión de los bienes y recursos naturales, lo que } \\
\text { incrementó en un } 20 \% \text { los ingresos de los pobladores } \\
\text { por la cría de erizos (Thryonomys swinderianus) y la } \\
\text { introducción de la apicultura. }\end{array}$ & $\begin{array}{l}\text { Movimiento Mundial } \\
\text { por } \\
\text { los Bosques } \\
\text { Tropicales } \\
\text { (WRM por sus siglas } \\
\text { en inglés), } \\
2004 \\
\end{array}$ \\
\hline $\begin{array}{l}\text { Indonesia: Pueblo Dayak en la } \\
\text { primera área protegida con manejo } \\
\text { conjunto }\end{array}$ & $\begin{array}{c}\text { Las poblaciones indígenas se } \\
\text { empoderaron de su territorio y manejaron } \\
\text { los recursos del bosque de una manera } \\
\text { sostenible al recuperar las áreas degradas } \\
\text { y } 1 \text { usar racionalmente los recursos para } \\
\text { su consumo. }\end{array}$ & $\begin{array}{c}\text { La reserva Parque Nacional Kayan Mentarang } \\
\text { presentaba restricción para los pobladores, quienes } \\
\text { estaban marginados y sumidos en la pobreza, y el } \\
\text { parque estaba en deterioro. El gobierno decidió el } \\
\text { empoderamiento de la población, lo cual mejoró su } \\
\text { calidad de vida. }\end{array}$ & Eghenter, 2003 \\
\hline $\begin{array}{l}\text { Tanzania: conocimiento tradicional } \\
\text { en la restauración de bosques }\end{array}$ & $\begin{array}{l}\text { El conocimiento tradicional de la } \\
\text { población Sukuma del lago Victoria } \\
\text { permitió restaurar sus bosques mediante } \\
\text { el sistema llamado ngitili. }\end{array}$ & $\begin{array}{c}\text { El ngitili favoreció la conservación de los suelos, } \\
\text { promoviendo la regeneración de la vegetación y la } \\
\text { reducción de la erosión, lo que contribuyó a mejorar la } \\
\text { producción agrícola y ganadera en la región, así como } \\
\text { la producción de madera para comercializar en el } \\
\text { mercado local. }\end{array}$ & WRM, 2004 \\
\hline $\begin{array}{l}\text { Restauración ecológica de los } \\
\text { bosques de la región Chanting } \\
\text { County, China }\end{array}$ & $\begin{array}{l}\text { Las autoridades ambientales, en conjunto } \\
\text { con las poblaciones locales, realizaron } \\
\text { labores de restauración ecológica de } \\
\text { bosques, lo que disminuyó la presión } \\
\text { generada sobre los ecosistemas y la } \\
\text { explotación excesiva de madera. }\end{array}$ & $\begin{array}{c}\text { Las labores de restauración contribuyeron a mejorar la } \\
\text { calidad de vida de los pobladores rurales. La erosión de } \\
\text { los suelos se redujo en } 37,7 \% \text {, lo que favoreció su } \\
\text { fertilidad. Adicionalmente, la humedad relativa } \\
\text { aumentó de } 55 \% \text { a } 63 \% \text {. Las condiciones sociales y } \\
\text { demográficas mejoraron, ya que la tasa de emigraciones } \\
\text { a otros centros poblados se redujo y mejoró la } \\
\text { producción agrícola. }\end{array}$ & $\begin{array}{l}\text { Wang, Yang y Zhang, } \\
2010\end{array}$ \\
\hline
\end{tabular}

Fuente: elaboración propia 
A partir de la revisión de las experiencias en otros contextos geográficos y sociales, se identificaron los siguientes factores, que, en concordancia con Gomá (2008), se consideran determinantes para que exista una transformación social a partir de los procesos de recuperación ecosistémica en las comunidades:

- Intervención de las poblaciones: la población tiene su propia fuerza reguladora e iniciativa que permite, desde el individuo hasta la colectividad, desarrollar conocimientos y habilidades de manera integral en la realidad social en la que está inmersa.

- Articulación Social: formación de canales de comunicación y relaciones entre diferentes miembros de la población para lograr un objetivo común.

- Articulación con instituciones del estado: formación de canales de comunicación y relaciones entre diferentes miembros de la población y las instituciones del estado con miras a contribuir al cumplimiento de un objetivo común.

- Beneficios económicos (incremento de la productividad): se refiere al incremento de la productividad de los ecosistemas para la producción de servicios ambientales, que repercute en el mejoramiento de las condiciones económicas de las personas.

- Beneficios sociales (incremento de la calidad de vida): se relaciona con los beneficios sociales suministrados por los ecosistemas en proceso de restauración ecológica.

- Aplicación de conocimientos locales: se refiere a la aplicación de conocimientos locales en las acciones de restauración ecológica.

- Empoderamiento de las poblaciones con su territorio: la población cobra autonomía en la toma de decisiones y logra ejercer control sobre sí mismos como grupo social y sobre su territorio (Ministerio de Agricultura y Desarrollo Rural [MADR], 2003).

\section{Presentación de la propuesta}

Teniendo en cuenta que a la degradación ecosistémica se le debe ver como un problema ambiental; y a la restauración ecológica, como una necesidad de superar esa degradación, es necesario que para plantear alternativas de solución más eficientes y acordes con la dinámica socioambiental de la zona, la restauración se vea desde un enfoque holístico que permee el sistema ambiental y sus dimensiones (ecológica, sociocultural, económica, política e institucional).

En este sentido, la propuesta metodológica busca que el proceso de restauración ecológica trascienda las diferentes dimensiones del medio ambiente y se integre en los saberes y cultura de las poblaciones. Por tal razón, se debe ver el proceso con un enfoque integrador que garantice el mantenimiento de la restauración a largo plazo y, por consiguiente, la recuperación de los ecosistemas degradados de los cuales dependen las poblaciones para su desarrollo. Así, ver la restauración ecológica como una construcción social del proceso resulta interesante, ya que constituye una nueva propuesta que impulsa el desarrollo sustentable en las poblaciones, dado que, a partir de la construcción de conocimiento, de canales de comunicación, de conciencia de su entorno natural, en fin, de la reconstrucción de la relación sociedad-naturaleza de una manera sana y equilibrada, es posible aprovechar las potencialidades de los ecosistemas para fomentar procesos de desarrollo sustentable.

\section{Principios generales del modelo metodológico.}

Se definieron nueve (9) principios generales a partir del análisis de la información bibliográfica, y la obtenida mediante las técnicas de recolección de datos desarrolladas a lo largo de la investigación: 
I. Flexibilidad y dinamicidad en las etapas del modelo: las acciones de restauración ecológica varían con las condiciones socioculturales, económicas, políticas y ecológicas del territorio. Así las cosas, el modelo debe adaptarse a estos cambios, por tanto, las fases del modelo son dinámicas en cuanto a duración, objetivos y productos.

II. Intervención de las poblaciones en el proceso de restauración ecológica: es fundamental, ya que el proyecto sólo puede ser exitoso a largo plazo si es socialmente aceptado, y si se trabaja conjuntamente con las comunidades en todas sus fases.

III. Articulación social al interior de la comunidad y fuera de ella: permitirá crear y fortalecer las organizaciones sociales y construir canales de comunicación.

IV. Incorporación de los procesos locales a la reflexión sobre políticas públicas nacionales y locales: son importantes para garantizar la sostenibilidad del proyecto de restauración.

V. Mejoramiento de las condiciones socioeconómicas de las personas y grupos sociales: los beneficios económicos deben ser evidentes en la planificación del proceso de restauración, es decir, traducidos en oportunidades locales de generación de ingresos.

VI. Mejoramiento de la calidad de vida de la población: fortalecimiento y reconstrucción del tejido social, cultural e intelectual de la población local (beneficios socioculturales).

VII. Inclusión de los conocimientos locales sobre el manejo y uso de los ecosistemas: dicha inclusión funciona como complemento al conocimiento científico.

VIII. Enfoque de manejo adaptable: el proceso de restauración ecológica debe ser flexible con el fin de responder a los cambios derivados de las dinámicas sociales, políticas, culturales y ecológicas del territorio.

IX. Autonomía de la población en la toma de decisiones: permite ejercer el control sobre sí misma como grupo social, y sobre su territorio.

\section{Exigencias básicas.}

Las exigencias básicas para la aplicación del modelo son las siguientes:

a) Contar con áreas de interés colectivo donde se pretenda desarrollar el proyecto de restauración ecológica.

b) Comprensión y disposición de la comunidad para involucrarse en las actividades concernientes al proyecto de restauración ecológica. Este proceso debe ser transparente y autónomo, y surge de la iniciativa y necesidades de la población.

c) Debe existir un marco legal e institucional que soporte el proceso de restauración ecológica.

d) Deben existir los recursos financieros necesarios para que las comunidades y el equipo investigador cuenten con los recursos para desarrollar el proyecto.

\section{Formulación del modelo.}

En la figura 4 se presenta el modelo propuesto de restauración como una estrategia de construcción social para el desarrollo sustentable en la vereda Chipautá, Guaduas (Cundinamarca). Cabe aclarar que este modelo es general y puede ser aplicado en otras comunidades y contextos. La experiencia en la vereda Chipautá permitió extraer algunos elementos que soportarán el modelo y complementarlo con las otras etapas de la investigación. 


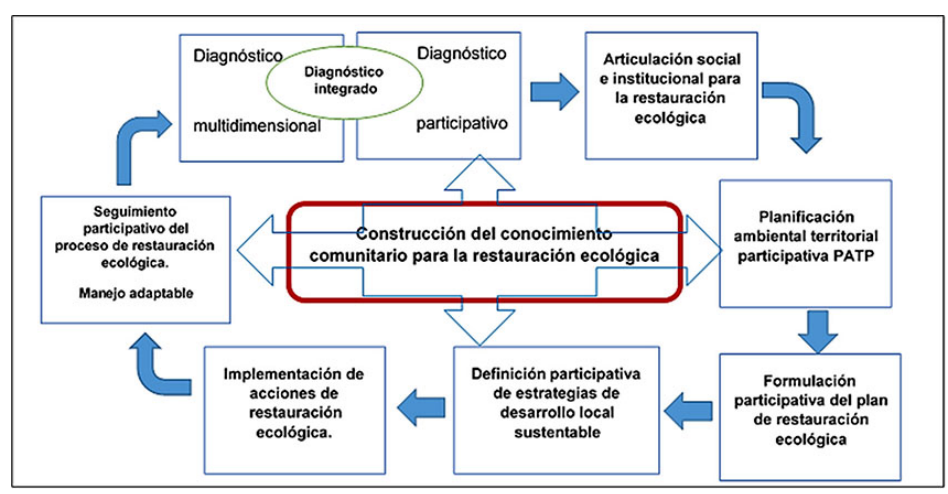

FIGURA 4 .

Modelo metodológico de restauración ecológica como estrategia de construcción social para el desarrollo sustentable propuesto para la vereda Chipautá, Guaduas (Cundinamarca). Fuente: elaboración propia

\section{Etapas del modelo}

\section{Diagnóstico integrado}

En esta etapa se pretende obtener información que soporte el plan de restauración ecológica desde una perspectiva multidimensional (sociocultural, político-normativo, económico y ecológico) y participativa.

\section{Construcción del conocimiento comunitario}

A partir de lo obtenido en el diagnóstico integrado es posible identificar los vacíos conceptuales o de conocimientos en la población, que son necesarios para complementar los conocimientos propios y, de esta forma, fomentar y fortalecer la intervención de la población en todo el proceso de restauración.

\section{Articulación social e institucional}

En los procesos de restauración ecológica es indispensable que exista una relación estrecha y estratégica entre las instituciones y la sociedad, dado que las instituciones fortalecen la población y facilitan su desarrollo.

\section{Planificación ambiental territorial participativa de la restauración ecológica}

La planificación del territorio en los procesos de restauración ecológica es fundamental, en el sentido que los diferentes actores sociales presentes en el área objeto de restauración interactúan en y con el territorio, el cual responde, en primera medida, a las necesidades socioculturales, políticas y económicas de la población (Lozano, 2008).

Formulación participativa del plan de restauración ecológica como una estrategia de construcción social para el desarrollo sustentable

En esta etapa se pretende, de acuerdo con lo encontrado en las fases anteriores, formular el plan de restauración ecológica. Tal actividad requiere la intervención de la población dentro del proceso con el fin de construir socialmente la restauración en torno a las necesidades, expectativas y actores sociales involucrados. 


\section{Definición participativa de estrategias de desarrollo local sustentable}

A partir del proceso de restauración ecológica surgen estrategias de desarrollo sustentable con el fin de buscar oportunidades de generación de ingresos en la población para incentivar la participación permanente. Dentro de las estrategias, es importante considerar el suministro de beneficios a corto plazo, dado que esto conduce a la aceptación de objetivos a largo plazo (Gann \& Lamb, 2006).

\section{Implementación de las acciones de restauración}

En esta fase, se procederá a la implementación de las acciones de restauración, en conjunto con la población local. Estas acciones están asociadas a los objetivos y metas propuestos en la fase de formulación.

\section{Seguimiento participativo al proceso de restauración}

El seguimiento participativo al proceso de restauración ecológica es importante y necesario para la continuidad del proceso a largo plazo, ya que en este, la población, en conjunto con los técnicos, realiza una revisión y un análisis periódico de la información que surge del proyecto.

\section{Validación del modelo por criterio de expertos}

El modelo metodológico propuesto se sometió a validación por criterio de expertos. En esta experiencia de investigación se solicitó la colaboración de los posibles candidatos adjuntando una invitación formal a participar como jueces validadores en la que se presentaba el modelo metodológico de restauración ecológica.

\section{Definición de criterios de validación}

Los objetivos de la investigación, y el objetivo específico de la validación por juicio de expertos, basados en la metodología propuesta por Skjong y Wentworth (2001), definen los siguientes criterios de validación, los cuales se comportan como categorías de análisis que agrupan las distintas preguntas realizadas a los expertos a partir del cuestionario diligenciado y su discusión. Posteriormente, se realizó el análisis de los datos obtenidos (tabla 5).

I. Conveniencia de incluir la dimensión social como elemento indispensable e integrador dentro de los procesos de restauración ecológica para lograr una construcción social.

II. Rigurosidad científico-técnica en la formulación del modelo metodológico.

III. Pertinencia del modelo metodológico dentro del área de conocimiento de la restauración ecológica. 
TABLA 5 .

Resultados del proceso de validación

\begin{tabular}{|c|l|}
\hline Criterio & \multicolumn{1}{c|}{ Resultados } \\
\hline I & $\begin{array}{c}\text { El modelo metodológico presentó una aceptación del } 90 \% \text { respecto a este criterio. } \\
\text { El } 100 \% \text { de los expertos considera importante la inclusión de las dimensiones de } \\
\text { los sistemas socioecológicos en el análisis e implementación de los procesos de } \\
\text { RE, la planificación ambiental territorial para la selección de las áreas a restaurar, } \\
\text { y que esta, a su vez, deba ser participativa. Además, coinciden en la pertinencia } \\
\text { de incorporar la construcción del conocimiento comunitario como elemento } \\
\text { transversal en el modelo. Uno de los expertos se muestra neutro ante esto último. }\end{array}$ \\
\hline II & $\begin{array}{l}\text { Todos los expertos (100\%) coinciden en que el modelo metodológico presentó, } \\
\text { para su formulación, una rigurosidad científico técnica importante, teniendo en } \\
\text { cuenta el trabajo realizado en campo y la validación de la información, pero } \\
\text { recomiendan la divulgación del modelo para identificar elementos que puedan } \\
\text { complementarlo y, posteriormente, llevarlo a su implementación. }\end{array}$ \\
\hline III & $\begin{array}{l}\text { El 100\% de los expertos considera pertinente el modelo metodológico dentro del } \\
\text { área de conocimiento de la restauración ecológica, dada la necesidad de contar } \\
\text { con un modelo que articule a los actores sociales con el proceso de restauración, } \\
\text { no como un elemento netamente participativo, sino como un factor estructurante } \\
\text { e interviniente que permita un diálogo de saberes que fortalezca el proceso y } \\
\text { garantice su éxito a largo plazo. } \\
\text { De igual manera, consideran que la existencia de un modelo de restauración } \\
\text { ecológica que lleve a una construcción social abre las puertas de un nuevo } \\
\text { paradigma de restauración que permita la reconstrucción de la relación entre las } \\
\text { comunidades y los ecosistemas de los cuales dependen. }\end{array}$ \\
\hline
\end{tabular}

Fuente: elaboración propia

\section{Discusión}

La ejecución de este proyecto, como proceso de investigación en la vereda Chipautá, aportó herramientas significativas frente al trabajo realizado en restauración ecológica, en el cual, en concordancia con lo planteado por Gann y Lamb (2006), Egan (2003) y OIMT (2002), se evidenció la necesidad de reconocer como actores principales del proceso a las comunidades que habitan los espacios naturales. De igual manera, es fundamental reconocer la participación comunitaria como estrategia de transformación y construcción social a favor del manejo sustentable de los ecosistemas, que facilita el trabajo conjunto entre la población y las instituciones del estado, así como la necesidad de tener en cuenta la importancia de la práctica dentro de un proceso dinámico y formativo que debe trascender de los contenidos teóricos a las acciones concretas que mitiguen el impacto causado a los ecosistemas y garanticen su recuperación a largo plazo. Lo anterior sin dejar de lado la realidad social, política, económica y cultural que interactúa constantemente con el entorno, y que define sus tendencias de transformación y aprovechamiento, las cuales necesariamente conducen a procesos de transformación social en una relación bidireccional.

De acuerdo con Subirats (2010) y Gomá (2008), la transformación social implica un cambio que debe experimentarse desde el interior de los procesos, las organizaciones y, fundamentalmente, de las personas, el cual está permeado por algunos elementos definitorios que parten de la existencia de un colectivo humano que es sujeto y protagonista de acciones y decisiones. De igual manera, este cambio contiene mecanismos y procesos de interacción que se representan en la reciprocidad mutua que, evidentemente, están ligados al arraigo a un territorio; un espacio geográfico que articula agentes, instrumentos y contenidos que condicionan 
su vida e incorpora significados de pertenencia. Lo anterior se integra con la necesidad de tener una visión sistémica del ambiente para el desarrollo con procesos de restauración ecológica en varias regiones del país y la instauración de cambios en la relación sociedad-naturaleza. En este sentido, las actividades de reforestación y recuperación de la cárcava sobre la Quebrada Llanadas en la vereda Chipautá, que aunque difieren de la instauración de un proyecto de restauración ecológica, permitieron evidenciar estos procesos de transformación social y de apropiación del territorio, los cuales aportaron elementos para la construcción de la propuesta metodológica y se vieron reflejados en los resultados de la triangulación procedentes de las diferentes fuentes de información.

Adicionalmente, los elementos determinantes del modelo encontrados a partir de la experiencia con la vereda Chipautá, concuerdan en campo con los principios planteados por las directrices de la OIMT (2002), y lo propuesto por Gann y Lamb (2006), referente a que en el proceso debe existir una intervención social de la población que garantice el éxito a largo plazo del proyecto, incorporando técnicas y conocimientos desde la comunidad. También, es necesario analizar, desde una visión integral, los procesos de restauración ecológica, incorporando variables sociales, políticas y económicas que influyan directamente sobre los procesos de degradación ambiental, y que repercutan en la calidad de vida de las poblaciones (OIMT, 2002).

\section{Conclusiones}

Mediante la revisión sistemática de bibliografía de experiencias de restauración, fue posible analizar la relación existente entre los procesos de restauración ecológica y la transformación social de las poblaciones asociadas a los ecosistemas degradados en proceso de restauración, lo cual permitió contribuir con la identificación de los factores condicionantes del modelo metodológico.

Las etapas propuestas en el marco metodológico de la investigación permitieron conjuntamente el diseño del modelo metodológico de restauración ecológica propuesto para la vereda Chipautá. Así mismo, la validación por criterio de expertos permitió disminuir el grado de sesgo en la investigación y confirmar la pertinencia del modelo en el campo académico de restauración ecológica.

La ejecución de este proyecto como proceso de investigación aportó herramientas significativas frente al trabajo realizado en restauración ecológica, en el cual se evidenció la necesidad de reconocer como actores principales del proceso, a las comunidades que habitan los espacios naturales. De igual manera, es fundamental reconocer la participación comunitaria como estrategia de transformación y construcción social a favor del manejo sustentable de los ecosistemas que facilita el trabajo conjunto entre la población y las instituciones del estado, así como la necesidad de tener en cuenta la importancia de la práctica dentro de un proceso dinámico y formativo que debe trascender de los contenidos teóricos a las acciones concretas que mitiguen el impacto causado a los ecosistemas y garanticen su recuperación a largo plazo. Lo anterior sin dejar de lado la realidad social, política, económica y cultural que interactúa constantemente con el entorno y define sus tendencias de transformación y aprovechamiento.

\section{Referencias}

Barkin, D. (2012). Communities Constructing Their Own Alternatives in the Face of Crisis. Mountain Research and Development, 32(1), 12-22. https://doi.org/10.1659/MRD-JOURNAL-D-11-00088.S1

Cantillo, E., Lozada, A., y Pinzón, J. (2009). Caracterización sucesional para la restauración de la reserva forestal Cárpatos, Guasca, Cundinamarca. Colombia Forestal, 12, 103-118.

Barrera-Cataño, J., Contreras-Rodríguez, S., Garzón-Yepes, N., y Moreno-Cárdenas, A. (2010). Manual de restauración ecológica de los ecosistemas disturbados del Distrito Capital. Bogotá: Secretaría Distrital de Ambiente y Pontificia Universidad Javeriana. 
Clewell, A., Rieger, J., y Munro, J. (06 de 2000). Guidelines for Developing and Managing Ecological Restoration Projects. Society for Ecological Restoration (SER). Recuperado de http://www.iucnsscrsg.org/STORAGE/RSG\%20C D/PDFs/SERGuide.pdf

Egan, D. (2003). Ecological Restoration and Sustainable Development. Ecological Restoration, 21(3), 161-162.

Gann, G., y Lamb, D. (2006). La restauración ecológica - un medio para conservar la biodiversidad y mantener los medios de vida. Recuperado de http://www1.inecol.edu.mx/repara/download/III_1_RestauracionEcologica_GannAn dLamb2006.pdf

Gomá, R. (2008). La acción comunitaria: transformación social y construcción de ciudadanía. Revista de educación social, 7. Recuperado de http://www.eduso.net/res/?b=10\&c $=90 \& \mathrm{n}=251$

Ministerio de Agricultura y Desarrollo Rural (MADR). (2003). Informe Final Proyecto Piloto de Zonas de Reserva Campesina: hacia una estrategia de la frontera de estabilización de la frontera agricola con desarrollo humano. Bogotá: IICA Biblioteca Venezuela.

Hallett, L., Hobbs, R., y Mooney, H. (2011). Intervention Ecology: Applying Ecological Science in the Twenty-first Century. BioScience, 61(6), 442-450.

Hernández, S., Fernández, C., y Baptista, P. (2004). Metodologia de la investigación. México: McGraw-Hill.

Keenleyside, K., N. Dudley, S. C., Hall, C., y Stolton, S. (2014). Restauración Ecológica para Areas Protegidas: Principios, directrices y buenas prácticas. Gland, Suiza: UICN.

Lozano, F. (Ed.). (2008). Herramientas de manejo para la conservación de la biodiversidad en paisajes rurales. Bogotá: Instituto de Investigación de Recursos Biológicos Alexander von Humboldt.

Organización Internacional de las Maderas Tropicales (OIMT). (2002). Directrices de la OIMT para la restauración, ordenación y rehabilitación de bosques tropicales secundarios y degradados. Recuperado de http://www.itto.int/di rect/topics/topics_pdf_download/topics_id=1540000\&no=3\&disp=inline

Parques Nacionales Naturales de Colombia (PNN). (2009). Recuperado el 23 de 04 de 2015, de http://www.parquesnacionales.gov.co/PNN/portel/libreria/pdf/AREAS_PROTEGIDAS_PAIS_2009_R EPORTADAS_PNN.pdf

Román, F., Levy, S., Aguirre, J., y Sánchez, A. (2011). Arboles de la selva Lacandona útiles para la Restauración Ecológica. México D.F.: Comisión Nacional Forestal.

Ritchlin, J. (2001). Healing the Land... Healing Ourselves: A Guide to Ecological Resources for British Columbia. Vancouver, BC: BC Environmental Network Educational.

Skjong, R., y Wentworth, B. (2001). Expert Judgment and Risk Perception. Proceedings of the Eleventh (2001) International Offshore and Polar Engineering Conference. Norway: The International Society of Offshore and Polar Engineers

Subirats, J. (2010). Los grandes procesos de cambio y transformación social. Recuperado de https://ddd.uab.cat/pub/ca plli/2010/119513/camsoccoosig_a2010p8.pdf

\section{Notas}

* Artículo de investigación, producto del trabajo La restauración ecológica como estrategia de construcción social en la vereda Chipautá, municipio de Guaduas, Cundinamarca. Fue financiado por el convenio marco entre Pacific Stratus Energy y la Universidad Distrital Francisco José de Caldas de Bogotá, Colombia.

\section{Licencia Creative Commons CC BY 4.0}

Cómo citar este artículo: Hernández, R., y Cantillo, E. (2018). La restauración ecológica como estrategia de construcción social en la vereda Chipautá, municipio de Guaduas, Cundinamarca, Colombia. Ambiente y Desarrollo, 22(42), 1-14. https://doi.org/10.11144/Javeriana.ayd22-42.reec 